Research Article

\title{
Towards an Application Helping to Minimize Medication Error Rate
}

\author{
Ali I. Alharbi $\mathbb{D},{ }^{1}$ Valerie Gay $\mathbb{D}^{2},{ }^{2}$ Mohammad J. AlGhamdi $\mathbb{D}^{3},{ }^{3}$ Ryan Alturki $\mathbb{D}^{3}$, \\ and Hasan J. Alyamani $\mathbb{C}^{4}$ \\ ${ }^{1}$ Department of Information Systems, King Abdulaziz University, Jeddah 215089, Saudi Arabia \\ ${ }^{2}$ School of Electrical and Data Engineering, Faculty of Engineering and Information Technology, University of Technology Sydney, \\ Sydney 2007, Australia \\ ${ }^{3}$ Department of Information Science, College of Computer and Information Systems, Umm Al-Qura University, Makkah, \\ Saudi Arabia \\ ${ }^{4}$ Department of Information Systems, Faculty of Computing and Information Technology, King Abdulaziz University, Rabigh, \\ Saudi Arabia
}

Correspondence should be addressed to Ryan Alturki; rmturki@uqu.edu.sa

Received 9 May 2021; Revised 14 June 2021; Accepted 21 June 2021; Published 28 June 2021

Academic Editor: Fazlullah Khan

Copyright (C) 2021 Ali I. Alharbi et al. This is an open access article distributed under the Creative Commons Attribution License, which permits unrestricted use, distribution, and reproduction in any medium, provided the original work is properly cited.

Medication errors related to medication administration done by both doctors and nurses can be considered a vital issue around the world. It is believed that systematisation and the introduction of main documents are done manually, which might increase the opportunities to have inaccuracies and errors because of unexpected wrong actions done by medical practitioners. Experts stated that the lack of pharmacological knowledge is one of the key factors, which play an important role in causing such errors. Doctors and nurses may face problems when they move from one unit to another and the medication administration list has changed. However, promoting public health activities and recent AI-enabled applications can provide general information about medication that helps both doctors and nurses administer the right medication. However, such an application can require a lot of time and effort to search and then find a medication. Therefore, this article aims to investigate whether AI-enabled applications can help avoid or at least minimize medication error rates.

\section{Introduction}

Public health has made a significant impact on health populations, making people healthy and saving lives. Therefore, healthcare remains one of the main areas of investment in AI-enabled applications and is rapidly evolving at present. Medication administration errors have become a serious patient safety issue due to the increased morbidity and mortality rates associated with such errors. According to the World Health Organization, medication errors (MEs) are conventionally defined as "any preventable event that may cause or lead to inappropriate medication use or patient harm while the medication is in the control of the health care professionals, patients, or consumers" [1]. Cohen and Williams defined MEs as "failure in the treatment process that leads to or has the potential to lead to, harm to the patient" $[2,3]$. The medication treatment process is generally a multistage process involving a multidisciplinary team. It commences with a doctor's prescription, followed by the pharmacist's check-up, and finally, the nursing staff administering the medication to the patient [4]. Medication administration is significantly one of the most complicated yet most vital processes of nursing care, which requires the right knowledge of the nursing tasks.

Similarly, Al-Harkan et al. stated that "implementation of medication orders is an important part of the process of treatment and care of patients and it is considered as a major component of a nurse's task, and in the meantime, patient safety has particular importance" [5]. One of the many challenges nurses face is when they transfer across units as 
the medication lists they use also change. This results in a lack of knowledge in medication details, including the right dose, right time, right route, interactions of medications, and contraindications [6]. Therefore, this project aims to assist nurses to reduce MEs by providing them with the Nurses Guide application. The nurses will have the opportunity to retrieve medication details in one easy-to-use application because the application displays the common medications for each unit and the interactions of the common medications with each other.

1.1. Problem Statement. According to the WHO, one of the factors associated with $\mathrm{ME}$ is insufficient medication knowledge and experience. Nurses spend approximately $40 \%$ of their time in hospitals administering medications to their patients [7]. The primary factor associated with MEs as perceived by nurses in the Kingdom of Saudi Arabia is that nurses are not familiar with the unit environment and the medications used in specific units $[8,9]$.

Many applications have attempted to solve these issues concerning medication knowledge by displaying medications in general, such as digitizing medication handbooks to provide comprehensive knowledge of medications. However, this solution provides excessive information, which was time-consuming. Furthermore, it does not fulfill the nurses' needs for a quick review of the most common medications used in a specific unit [5]. Another solution was adding a feature that categorizes the medication into top general common medications or according to a specific disease and not for units or conditions requiring a quick reaction [6].

1.2. Proposed Solutions. The problems mentioned above show the lack of proper applications that focus on providing the nurse with the right medication information in a shorter period. Therefore, we propose a solution that helps nurses prevent ME when being transferred from one unit to another by providing prompt feedback on the requested medication. This application provides the most commonly used medication in different units.

\section{Background and Related Work}

2.1. Background Information. Although hospitals vary widely in the services they offer, some may have multiple units, such as intensive care unit, emergency unit, neurology, and cardiology, and they provide nursing care for all patients with varying severity and illnesses. Several studies have revealed that patient and nurse have different definitions. A patient is "a person receiving or registered to receive medical treatment" [3], while a nurse can be defined as "a caregiver for patients, and assistant to manage physical needs, prevent illness and treat health conditions" [10]. Therefore, a nurse has to fulfill many tasks, including administering treatments and medications, collaborating with the team to plan for patient care, and recording medical history and symptoms [10]. According to a definition provided by the United States National Coordinating Council, "a medication error is any preventable event that may cause or lead to inappropriate medication use or patient harm while the medication is in the control of the health care professional, patient, or consumer. Such events may be related to professional practice, health care products, procedures and systems, including prescribing, order communication, product labeling, packaging, and nomenclature, compounding, dispensing, distribution, administration, education, monitoring, and use" $[11,12]$. MEs are associated with an increase in the number of medications for the elderly and children, including specific and general medications. Furthermore, according to the WHO, one of the factors associated with MEs is insufficient medication knowledge and experience [13].

Most of the medications are administered by nurses and nursing students; therefore, most MEs are common among them $[14,15]$. Nurses spend approximately $40 \%$ of their time in the hospital administering medicines to their patients [16]. Koohestani and Baghcheghi found that there are currently more than 20 thousand types of drugs in the world, and despite their therapeutic effects, they can have potential side effects with their specific instructions. Taken together, the results suggest that it is crucial for nurses and nursing students to pay attention to the necessary information about drugs in order to prevent the potential dangers of ME [17]. Furthermore, the findings from the study of Gorgich and his colleagues suggested that common MEs in medication prescription can affect incorrect medication concentration, incorrect-time medication administration, medication overdose, and incorrect way of medication administration [18]. However, there is some evidence to suggest that MEs by nursing professionals are high, yet the rate of error reporting among them is low [19]. According to a recent study by Marznaki et al., the rate of MEs in nursing staff was reportedly $50 \%$, and the most common type of MEs was forgetting medication prescriptions and incorrect dosage [20].

Another report revealed that hospital staff nurses working at night duty shift often lack experience and interruption could cause high MEs rates [21]. Although 12hour shifts usually start at 7 p.m. and end at 7 a.m., some shifts start at 3 a.m. and end at 3 p.m. Some scheduled shifts that may last longer than 8 or even 12 hours may not follow the traditional pattern of the day, evening, and night shifts [19]. Several studies showed the quantity of MEs being still high [22-24]. A study shows that nurses spend almost $45 \%$ of their work time performing medication therapy, with studies confirming that $59 \%-78 \%$ of MEs are associated with hospital staff nurses [23-26]. A Swedish study identified that almost $45 \%$ of MEs rates are related to wrong dosage and other errors [27]. Another study from Mexico showed that almost $45 \%$ of MEs involved administration and prescriptions errors [28]. These examples demonstrate that MEs are a critical global issue. In addition, MEs lead to significant clinical and financial consequences [29-31].

There are many factors associated with MEs. The primary factor of MEs as perceived by the nurses in the Kingdom of Saudi Arabia is that nurses are not familiar with the unit environment and the medications used in the unit [32]. Although nurses are knowledgeable in their small area 
of practice, they flounder outside this environment. In other words, when nurses are transferred across units, they do not know the medications most commonly used in the new unit because of the lack of pharmacological knowledge [33]. Alsulami et al. and his colleagues, who studied MEs in 15 Middle Eastern countries, found that the most common types of prescribing errors reported were incorrect doses with an incidence rate ranging from $0.15 \%$ to $34.8 \%$ of prescriptions, wrong frequency, and wrong concentration. Therefore, one of the most related factors for MEs is a lack of knowledge of medications [34]. Other studies showed a substantial positive effect of the number of years of experience on the average score obtained by nurses $[6,12,26,35-37]$.

Significantly different scores were obtained by nurses of different ranks, with senior staff nurses achieving higher scores. However, it is an expected outcome as senior staff nurses have more years of experience and more opportunities for formal or self-directed learning than junior nurses. This is supported a study by O'Shea that demonstrated that increasing seniority was associated with more medicationrelated activities and hence more experience in medication management [38]. Nevertheless, the existing imbalance of knowledge has to be managed because junior nurses are also involved in medication management [39] (Figure 1).

2.2. Related Work. We found many applications trying significantly to solve the nurse's lack of medication knowledge by displaying the medications in general. This is done by digitizing medication handbooks and providing comprehensive knowledge. However, this solution provides too many details, which would be time-consuming. Furthermore, it does not fulfill the nurses' needs to quickly review the most common medications in a specific unit. Another solution is adding features that would categorize the medications into top general common medications or according to a specific disease and not by unit or conditions that require a quick reaction. These previous applications have helped us think about a solution that helps nurses avoiding MEs. Therefore, we propose a solution where MEs are reduced when they move from one unit to another by providing prompt feedback on the requested medications.

Our application provides the most commonly used medications in different units. It is an application that helps improve the quality of care for patients to avoid errors related to pharmacological knowledge. When nurses move from one unit to another unit, they may face a problem when they do not have enough information and need to refer to a medication guide that provides information about the dosage, time, route, allergy, and interactions. Our proposed application will provide an effective and efficient way for displaying medication information to the nurse.

\section{Methodology}

The following study intends to include most of the current and available knowledge regarding published work about MEs in Saudi Arabia. The inclusion criteria incorporated in this study are the articles published in English but originated from Saudi Arabia. Articles that described or proposed the concept of medication errors in Saudi Arabia were also included in the study. The exclusion criteria were articles originating from Saudi Arabia but not primarily focusing on medication errors in Saudi Arabia. Articles and studies that are concerned with health-related geographical information systems were not included in the study. A total of four databases were used for research by this study, including PubMed. Keywords that were searched for included Medication Errors, Nurses students, Saudi Arabia, and telemedicine. After reviewing articles against the inclusion and exclusion criteria, the resulting outcome was 20 articles. A total of 243 articles were initially found using PubMed when the researcher first searched for medication errors. However, after the exclusion and inclusion criteria, the number of articles was eventually narrowed down to 3 . The research was also composed of advanced research for metadata only and focused on entering each keyword in the field. After reviewing all articles, only three met the criteria and this is the total number of articles that were retrieved for this research purpose [40].

All the materials and methods sections in these articles contained sufficient detail; we went through the procedures used to make sure they are not repeated. Materials can be divided into headed subsections if several methods are described.

\section{System Analysis}

In a research framework, the research strategy or methodology is used in the research process to assist in the investigation of a particular problem and to answer research questions more systematically. There are various research strategies, such as experimental studies or case studies, which can be utilised in diverse kinds of research. Nevertheless, none of the strategies is inherently superior. Rather, the context in which the strategy is used and its ability to answer particular research questions gives it a certain superiority. While there are different strategies, researchers are able to utilise a strategy within another strategy. For example, a study that involves a case study strategy can include an experimental strategy included [41].

The selection among various strategies can be guided or influenced by the philosophy, approach, and the kind of questions that a study aims to answer. One must also consider the data, time, resources, and tools availability, ethical issues, and the expertise of the researcher. In the next section, we will introduce the research strategies that are suitable for this study.

4.1. Requirement Specification. This section defines the functional and nonfunctional requirements that the application should fulfill, describes how the application should work, defines the boundaries and constraints of the application, and conducts a specification and requirements analysis. We concluded academic studies to confirm the nurses' need for this application. The steps of scientific 


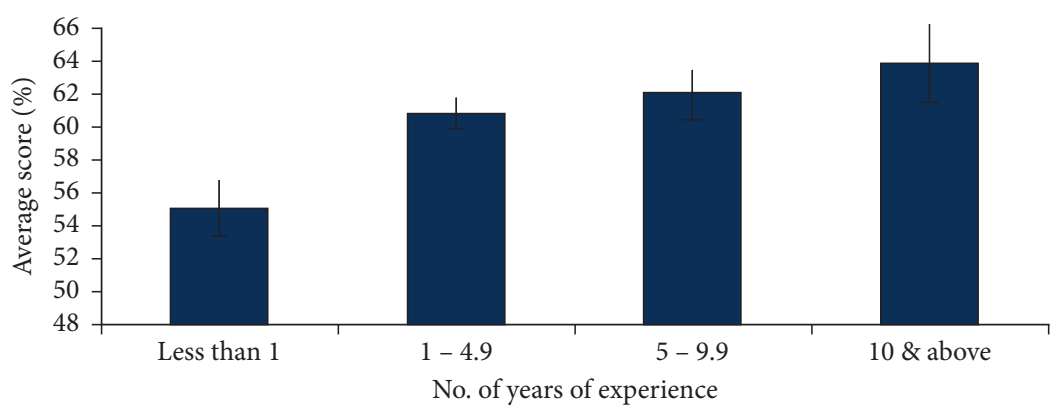

Figure 1: Average score and years of experience.

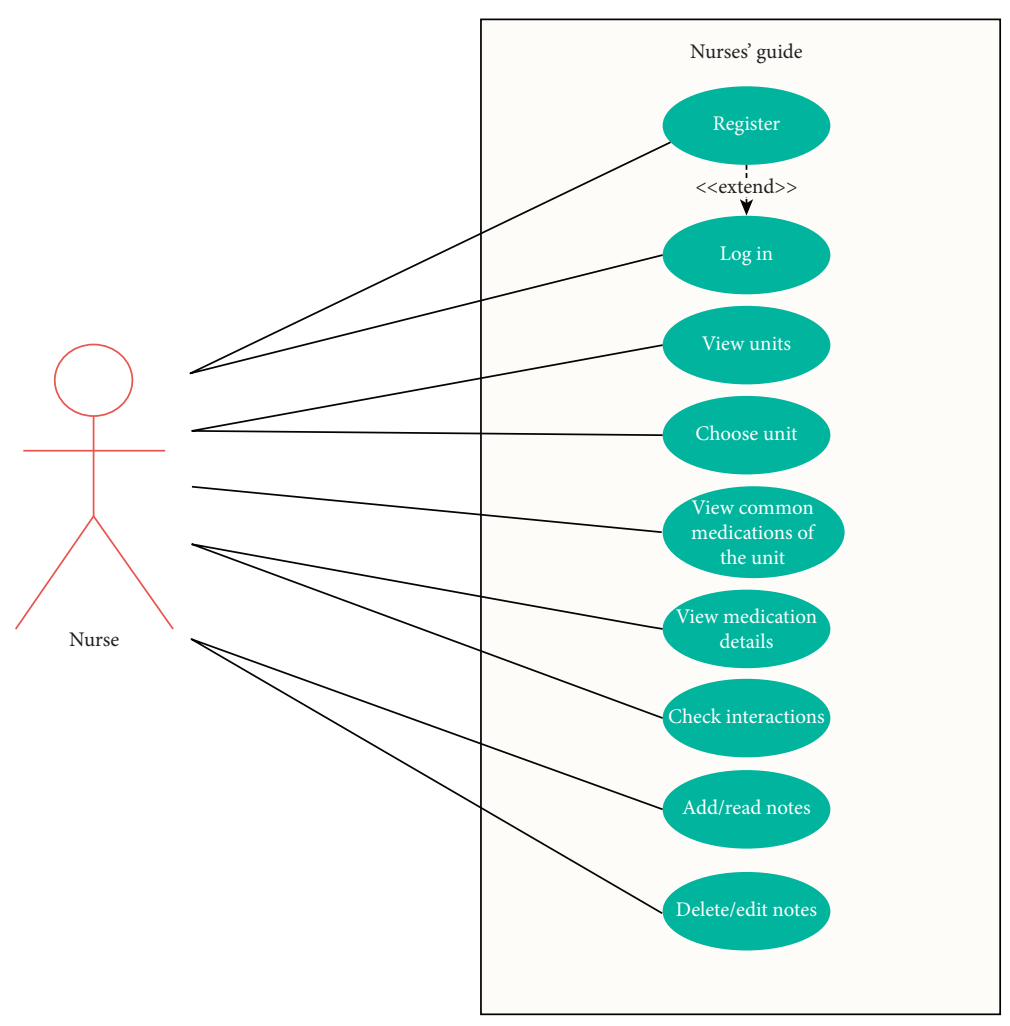

Figure 2: Use case diagram.

research were followed to discover the problems nurses face when they move from one department to another; however, enough information was not obtained after conducting the research and detailed figures on the amount of errors made by nurses were not found.

When giving medications, nurses should remember "five rules" and take them into account all the time: the right medication, the right patient, the right dose, the right time, and the right way [5]. Among the main causes of medication errors is exposure to distraction. Therefore, nurses should make sure that users have the least distractions when giving medications and that they are confident that they are ready to do so. Also, they should feel free to ask questions about a particular medication if they are in doubt and remember to check any medication before giving it to the patient. Therefore, the "Nurses' Guide" program has been implemented; refer to them as needed in the shortest possible time.

4.2. Requirements Analysis. Use case is UML Diagrams, a technique used for capturing functional requirements of a system. Typical interactions between users of a system and the system itself are described [42] (Figure 2).

This diagram illustrates the use case representing all of the possible functions that will occur in the application. Endusers are nurse and system. The sequence diagram illustrates how objects interact with each other, showing time ordering of interactions [40] (Figure 3).

This diagram illustrates that nurse can log in to the application by inserting her e-mail, ID, and password. Then by obtaining the nurses' previous data, there will be a 


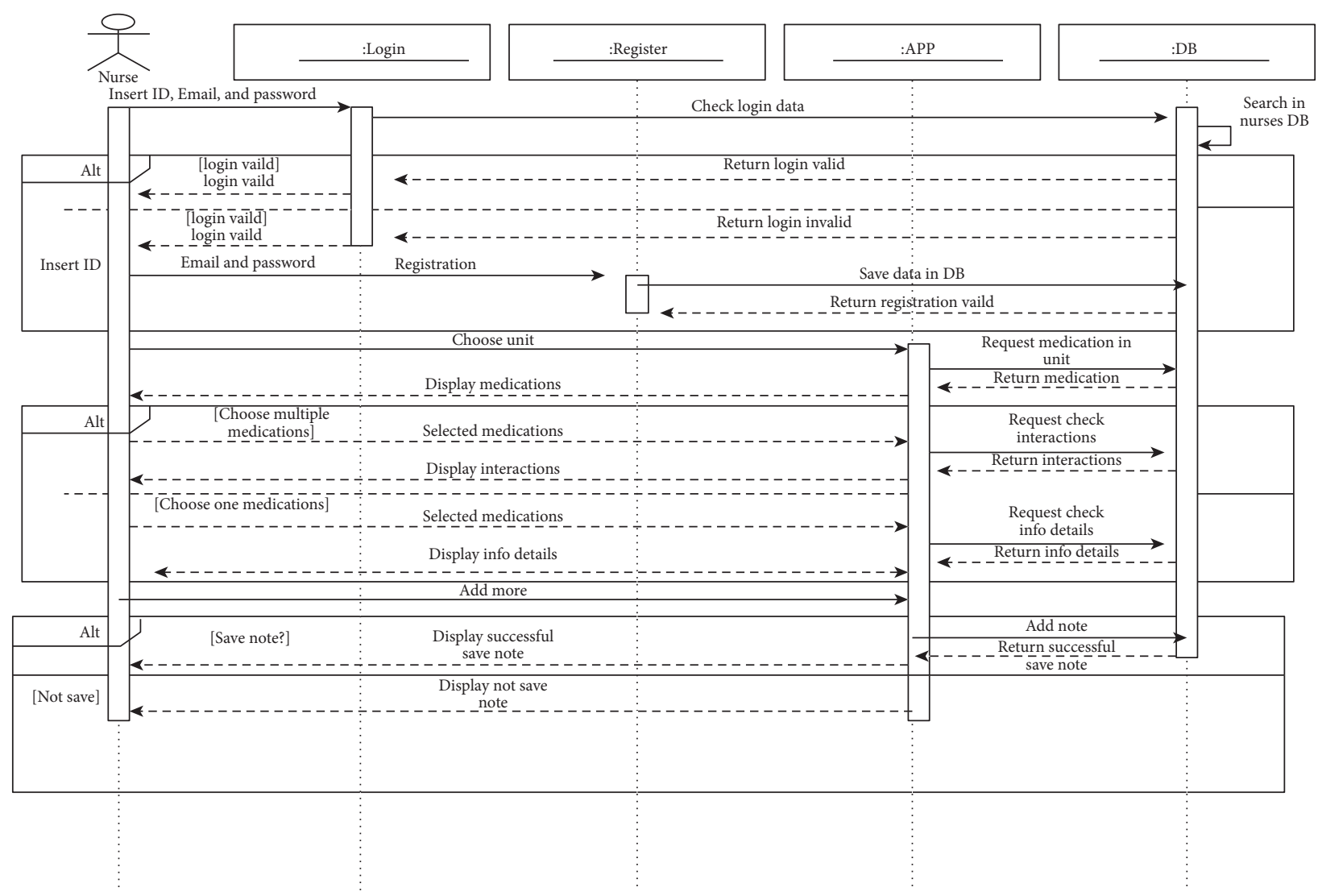

Figure 3: Sequence diagram.

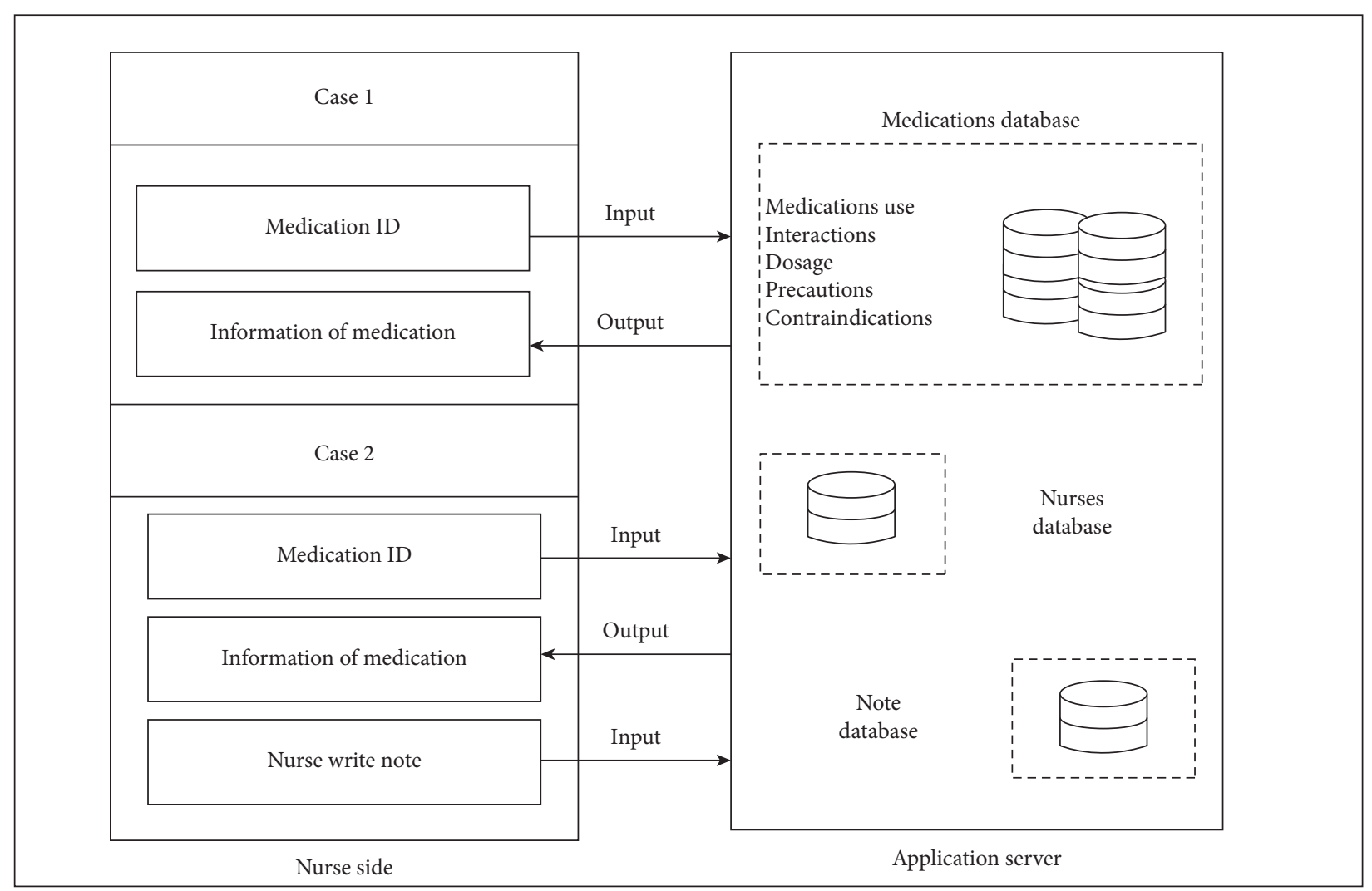

FIgURE 4: Conceptual design of the Nurses' Guide application. 
comparison between the inserted e-mail and password and the e-mail and password from the DB to check validity. If the login is invalid, it means the information inserted is incorrect or the nurse does not have an account. The nurse can register by creating a new account. Then, the nurse can choose one of the units displayed. After that, the nurse is able to view the common medications of that unit by choosing one of the medications to show its information or choosing multiple medications to show interactions. In addition, the nurse can add/read notes and the nurse can save notes or cancel.

\section{System Design}

The application is designed to display critical information about the most common medications in each unit in a hospital, reducing the amount of time and effort needed to look up medication details or check for interactions, enhance patient safety, and reduce medication errors. The nurse has an option to check medication interactions or dosage, route, time, precautions, side effects, and contraindications. Moreover, the nurse has an option to add notes in medications (Figure 4).

The Nurses' Guide system involves the following.

Application server: the server contains notes, interaction databases, and medication databases that act as a trusted source for providing the required data that a nurse needs during medication administration. It contains the following:

(1) Common medications for every unit, including the important information of time, dosages, precautions, contraindications, medication allergies, and route.

(2) Nurses notes, including the comments and any notes on the medications.

(3) Medication-medication interactions information.

(4) Nurses' accounts and data, including passwords, ID, and e-mails. Since our study scope focuses on the medication errors stage, the primary end-user is the nurse, while the secondary end-users are the healthcare provider (physician and pharmacist).

The Nurses' Guide application contains the following functions: the unit, the common medications, the information about medications, the interactions, and notes written by nurses.

After the nurse logs in to the Nurses' Guide application and chooses a certain unit, the system works in the following process, respectively: displaying the information of medication, displaying interactions, and displaying notes written by nurses.

\section{Conclusion}

Unfortunately, nurses can face several difficulties in getting the correct patient's information, resulting in medication errors related to their administration duties. This study proposes an AI-enabled application that focuses on the common medications used in each unit. The process of preventing medications errors can be supplemented by the introduction of a patient history using electronic means and other technologies. This helps the nurses access the required medication information in a very short period of time. Our proposed application will provide an effective and efficient way of displaying medication information to the nurse. Future work will involve performing usability testing and getting potential users' feedback and recommendations in order to improve the quality of the app.

\section{Data Availability}

The data used to support the findings of this study are available from the corresponding author upon request.

\section{Conflicts of Interest}

The authors declare that they have no conflicts of interest.

\section{References}

[1] World Health Organization, Medication Errors, World Health Organization, Geneva, Switzerland, 2016.

[2] M. R. Cohen, Medication Errors, American Pharmacists Association, Washington, DC, USA, 2007.

[3] D. J. P. Williams, "Medication errors," Journal-Royal College of Physicians of Edinburgh, vol. 37, no. 4, p. 343, 2007.

[4] P. Aspden and P. Aspden, Preventing Medication Errors, , pp. 409-46, National Academies Press, 2007.

[5] A. Al-Harkan, N. Al-Harkan, A. Al-Najjar, A. Al-Hunti, A. Al-Rashidi, and A. Al-Themery, "Investigation of medication errors in a tertiary care hospitals in the qassim region, Saudi Arabia," Open Access Macedonian Journal of Medical Sciences, vol. 8, no. E, pp. 209-212, 2020.

[6] R. F. Al-Ahmadi, L. Al-Juffali, S. Al-Shanawani, and S. Ali, "Categorizing and understanding medication errors in hospital pharmacy in relation to human factors," Saudi Pharmaceutical Journal, vol. 28, 2020.

[7] A. N. Isaacs, K. Ch'ng, N. Delhiwale, K. Taylor, B. Kent, and A. Raymond, "Hospital medication errors: a cross sectional study," International Journal for Quality in Health Care, vol. 33, 2020.

[8] H. H. Almutary and P. A. Lewis, "Nurses' willingness to report medication administration errors in Saudi Arabia," Quality Management in Health Care, vol. 21, no. 3, pp. 119-126, 2012.

[9] E. A. A. Hashish, W. A. Aljuaid, and O. Almuzaini, "Saudi nursing students attitudes towards patient safety and the influencing factors. A quantitative and qualitative study at the college of nursing-jeddah," International Journal of Nursing Education and Research, vol. 8, no. 1, pp. 53-66, 2020.

[10] Z. M. Ala'a, I. A. Aljasser, and B. Sasidhar, "Barriers to reporting medication administration errors among nurses in an accredited hospital in Saudi Arabia," Journal of Economics, Management and Trade, vol. 11, pp. 1-13, 2016.

[11] S. Ali, N. I. Aboheimed, I. A. Al-Zaagi, and D. S. Al-Dossari, "Analysis of medication errors at a large tertiary care hospital in Saudi Arabia: a retrospective analysis," International Journal of Clinical Pharmacy, vol. 39, no. 5, pp. 1004-1007, 2017.

[12] Y. A. Alomi, S. J. Alghamdi, and R. A. Alattyh, "National medication errors reporting system at ministry of health in Saudi Arabia," Pharmacology, Toxicology and Biomedical Reports, vol. 5, no. 1, 2019. 
[13] H. Al-Otaibi, S. A. Moawed, and M. F. Al-Harbi, "Nurses' medication errors in the ediatric emergency departme in Saudi Arabia," Middle East Journal of Nursing, vol. 101, no. 5829, pp. 1-11, 2018.

[14] M. Tobaiqy and D. Stewart, "Exploring health professionals' experiences of medication errors in Saudi Arabia," International Journal of Clinical Pharmacy, vol. 35, no. 4, pp. 542545, 2013.

[15] M. M. Abdel-Latif, "Knowledge of healthcare professionals about medication errors in hospitals," Journal of Basic and Clinical Pharmacy, vol. 7, no. 3, p. 87, 2016.

[16] A. I. Demehin, O. O. Babalola, and W. O. Erhun, "Pharmacists and nurses perception of medication errors in a Nigerian University teaching hospital," International Journal of Health Research, vol. 1, no. 2, pp. 51-61, 2008.

[17] H. Koohestani and N. Baghcheghi, "Investigation medication errors of nursing students in Cardiac Care Unite," Scientific Journal of Forensic Medicine, vol. 13, no. 48, pp. 249-255, 2008.

[18] E. A. C. Gorgich, S. Barfroshan, G. Ghoreishi, and M. Yaghoobi, "Investigating the causes of medication errors and strategies to prevention of them from nurses and nursing student viewpoint," Global Journal of Health Science, vol. 8, no. 8, p. 220, 2016.

[19] W. Alharbi, J. Cleland, and Z. Morrison, "Exploring healthcare professionals' perceptions of medication errors in an adult oncology department in Saudi Arabia: a qualitative study," Saudi Pharmaceutical Journal, vol. 27, no. 2, pp. 176-181, 2019.

[20] Z. H. Marznaki, S. Pouy, W. J. Salisu, and A. E. Zeydi, "Medication errors among Iranian emergency nurses: a systematic review," Epidemiology and Health, vol. 42, 2020.

[21] A. P. Montgomery, A. Azuero, M. Baernholdt et al., "Nurse burnout predicts self-reported medication administration errors in acute care hospitals," Journal for Healthcare Quality, vol. 43, no. 1, pp. 13-23, 2021.

[22] Z. Al-Zain and A. Althumairi, "Awareness, attitudes, practices, and perceived barriers to medical error incident reporting among faculty and health care practitioners (HCPs) in a dental clinic," Journal of Multidisciplinary Healthcare, vol. 14, pp. 735-741, 2021.

[23] A. Alnasser, I. Aldeeri, W. Aljamal, K. Sharahili, and Y. Alturki, "Patients' knowledge, awareness, and attitude regarding patient safety at a teaching hospital, Riyadh, Saudi Arabia," Journal of Family Medicine and Primary Care, vol. 9, no. 10 , p. 5236, 2020.

[24] T. M. Alshammari, K. A. Alenzi, Y. Alatawi, A. S. Almordi, and A. F. Altebainawi, "Current situation of medication errors in Saudi Arabia: a nationwide observational study," Journal of Patient Safety, vol. 17, 2020.

[25] A. Salam, D. M. Segal, M. A. Abu-Helalah et al., "The impact of work-related stress on medication errors in Eastern Region Saudi Arabia," International Journal for Quality in Health Care, vol. 31, no. 1, pp. 30-35, 2019.

[26] S. M. Alshahrani, K. M. Alakhali, and Y. M. Al-Worafi, "Medication errors in a health care facility in southern Saudi Arabia," Tropical Journal of Pharmaceutical Research, vol. 18, no. 5, pp. 1119-1122, 2019.

[27] K. S. Björkstén, M. Bergqvist, E. Andersén-Karlsson, L. Benson, and J. Ulfvarson, "Medication errors as malpractice-a qualitative content analysis of 585 medication errors by nurses in Sweden," BMC Health Services Research, vol. 16, no. 1, p. 431, 2016.
[28] M. A. Ramirez-Camacho, N. J. L. AKÉ, A. Gloria, and J. C. Torres-Romero, "Medication errors of intravenous therapy in the neonatal intensive care unit of a second-level hospital in southeastern Mexico," Latin American Journal of Pharmacy, vol. 39, no. 3, pp. 604-611, 2020.

[29] D. Horn, "Planning for the inevitable: medication errors," Pharmacy Today, vol. 26, no. 7, pp. 41-55, 2020.

[30] A. Salar, F. Kiani, and N. Rezaee, "Preventing the medication errors in hospitals: a qualitative study," International Journal of Africa Nursing Sciences, vol. 13, Article ID 100235, 2020.

[31] N. Patel, M. Desai, S. Shah, P. Patel, and A. Gandhi, "A study of medication errors in a tertiary care hospital," Perspectives in Clinical Research, vol. 7, no. 4, p. 168, 2016.

[32] M. Asad, "Assessment of nurses' knowledge for possible occurrence of medication errors in Riyadh province, Saudi Arabia," Asian Journal of Nursing Education and Research, vol. 5, no. 2, pp. 191-198, 2015.

[33] H. M. Dibbi, H. F. Al-Abrashy, W. A. Hussain, M. I. Fatani, and T. M. Karima, "Causes and outcome of medication errors in hospitalized patients," Saudi Medical Journal, vol. 27, no. 10, pp. 1489-1492, 2006.

[34] Z. Alsulami, S. Conroy, and I. Choonara, "Medication errors in the Middle East countries: a systematic review of the literature," European Journal of Clinical Pharmacology, vol. 69, no. 4, pp. 995-1008, 2013.

[35] M. Alshaikh, A. Mayet, and H. Aljadhey, "Medication error reporting in a university teaching hospital in Saudi Arabia," Journal of Patient Safety, vol. 9, no. 3, pp. 145-149, 2013.

[36] A. E. Aboshaiqah, "Nurses' perception of medication administration errors," American Journal of Nursing Research, vol. 2, no. 4, pp. 63-67, 2014.

[37] D. S. A. Dossari, M. I. Alnami, and N. A. Qureshi, "Analysis of reported E-prescribing medication administration errors at king saud medical city, riyadh: a cross-sectional, retrospective study," Journal of Pharmaceutical Research International, vol. 32, pp. 34-45, 2020.

[38] E. O'Shea, "Factors contributing to medication errors: a literature review," Journal of Clinical Nursing, vol. 8, no. 5, pp. 496-504, 1999.

[39] T. Kazaoka, K. Ohtsuka, K. Ueno, and M. Mori, "Why nurses make medication errors: a simulation study," Nurse Education Today, vol. 27, no. 4, pp. 312-317, 2007.

[40] A. A.-S. Al-Btoush, "Extracting entity relationship diagram (ERD) from English sentences," International Journal of Database Theory and Application, vol. 8, no. 2, pp. 235-244, 2015.

[41] R. Ibrahim, "Formalization of the data flow diagram rules for consistency check," 2010, http://arxiv.org/abs/1011.0278.

[42] A. Pyster, D. H. Olwell, N. Hutchison, S. Enck, J. F. Anthony Jr., and D. Henry, "Reliability, availability, and maintainability," Guide to the Systems Engineering Body of Knowledge (SEBoK), vol. 1, 2012. 